\title{
Designing Variable Ackerman Steering Geometry for Formula Student Race Car
}

gautampuneet20.pg@gmail.com

Shubham Sahai

shubhamdm097@gmail.com

Sachin Sunil Kelkar sachin.kelkar1299@gmail.com

Prajwal Sanjay Agrawal Corresponding Author agrawalprajwa1007@gmail.com ORCID: https://orcid.org/00000002-2847-7885

Mallikarjuna Reddy D dmreddy@vit.ac.in

School of Mechanical Engineering, Vellore Institute of Technology, Vellore, 632014, Tamil Nadu, India
Abstract - This paper describes the different types of steering mechanisms and benchmarks a method for the selection of a steering system. It also explains the step-bystep method of designing a variable Ackerman steering geometry. The research uses the knowledge of Ackerman and trapezoidal systems, turning radius estimation, steering effort calculation, and the RMS error tool to design and justify the policies selected. The result comprises a detailed flow for designing a variable Ackerman steering geometry along with a set of Matlab codes that are required for the calculation of turning radius, space, angles on inner and outer wheels, and many other parameters. This paper offers essential knowledge for those who are new to the field and an overview for those interested in steering design.

Keywords - Ackerman Steering, Steering geometry, Design, Formula student, RMS error.

\section{INTRODUCTION}

Formula SAE is an Engineering Design competition held by SAE International, which challenges students to build a formula-style race car evaluated in terms of engineering, performance, and efficiency. When it comes to high performance, vehicle dynamics plays a vital role in achieving the best possible outcomes. Along with mathematical modeling, good suspension and steering geometries are of utmost responsibility for the performance of the car. Since steering alone is responsible for maneuvering, so the selection and designing of a sound steering system become necessary for any car to be even drivable. Especially for land vehicles, the steering mechanism not only controls the maneuvering but also

Technical Article - Peer Reviewed

Published online - 13 Feb 2021

(C) 2021 RAME Publishers

This is an open access article under the CC BY 4.0 International License https://creativecommons.org/licenses/by/4.0/

Cite this article - Puneet Gautam, Shubham Sahai, Sachin Sunil Kelkar, Prajwal Sanjay Agrawal, Mallikarjuna Reddy D, "Designing Variable Ackerman Steering Geometry for Formula Student Race Car", International Journal of Analytical, Experimental and Finite Element Analysis, RAME Publishers, vol. 8, issue 1, pp. 1-11, 2021.

https://doi.org/10.26706/ijaefea.1.8.20210101 affects the suspension characteristics in motion. Thus, the selection of a correct mechanism is as important as designing and manufacturing vehicles [1]. In this paper, we will study the design of the steering mechanism. Firstly, to know the steering system is classified into three main types based on the mechanisms used. (As depicted in Figure 1)

i. Ackerman or Pro Ackerman Steering mechanism

ii. Parallel Steering mechanism

iii. Anti-Ackerman or Reverse Ackerman Steering mechanism

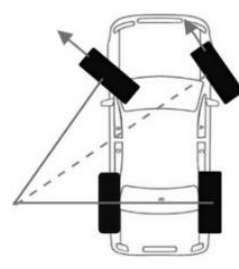

Ackerman

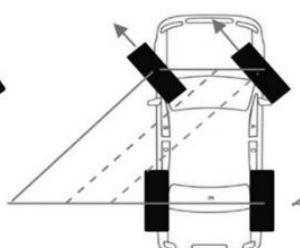

Parallel

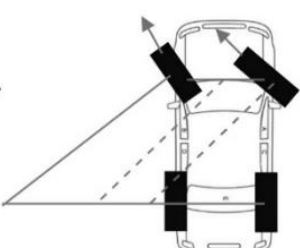

Reverse
Figure 1: Figure for the types of Steering Mechanism

As we know that the car is a rigid body and for it to make a turn, all the tires should make the turn around the same center (As seen in Figure 1), or else the tires will either push or pull each other. This push or pull effect will force the tires to change their positions from the desired path by creating a scrub, and it will also result in momentum loss for the car. 
So, to avoid this and help the car follow the pattern the above steering mechanisms are used based on different applications as each mechanism have their pros and cons.

Firstly, the Ackerman systems are relatively differentiated based on the amount of tire scrub (i.e., produced by each mechanism while the car is taking a turn). There is no scrub in the Ackerman system; the scrub effect increases in parallel mechanisms and increases more in the anti-Ackerman systems. Also, the appropriate steering angle required for a turn is always a function of wheel load, road condition, turn speed (depends on turn radius), and tire characteristics. Since the car witnesses both high speed and low-speed corners, so there is no ideal steering mechanism possible unless controlling the steering angle for all the wheels is carried out independently.

The selection of Ackerman, Parallel, or Anti-Ackerman geometry depends on the vehicle's cornering velocity during the turn. The cornering speed decides the amount of load transfer, further affecting the significance of slip angles on the car. As the slip angles have a higher impact at higher loads, it leads to the creation of lateral force accordingly [2]. The cornering velocity is directly proportional to the load transfer. Also, a slight difference in slip angles doesn't create a significant impact on the performance (lateral force) of the car at similar loads. So, in low-speed corners, the Ackerman mechanism is preferred to prevent the momentum loss caused by the wheels' scrubbing. Whereas when the car is going in a high-speed turn, due to high load transfer, maintaining an adequate slip angle on the wheels becomes more important than preventing the tire scrub, and hence parallel or anti-Ackerman is preferred in this case. Thus the system decision is based on the vehicle capacity and cornering conditions of the race.

To study the steering geometry firstly in 2011 Lili and co-workers tried to produce an Ackerman geometry system, but this process was limited to the calculation of the length of parts like the tie rod and it did not compare the performance [3]. Later in 2016 Malu and co-workers directly elaborated the effect of the steering moment arm angle and also used an iterative method for its prediction [4].
Then, in a study from Malik and co-workers from 2017 a discussion was carried out on suspension parameters that affect the steering characteristics but the limitation of this study was not discussing the steering parameters [5]. Then in the same year Biswal and co-workers designed the suspension parameters and steering types still this study lacked in the determination of the steering parameters [6]. Again, in the same year, Raut and co-workers threw light on energy transfer systems and suspension parameters and have not discussed the steering parameters [7]. Afterward, in 2018 Gitay and co-workers discussed the value of the parameters again, stating the iterative method for its vale prediction [8]. Lastly, in late 2018 Naveen and co-workers elaborated on the types of the steering system and the basis of its selection, but again a method for steering parameters goes missing [9]. So, from this, it was clear that a precise flow with the correct methods to find the steering parameters have always been missing, which is one of the main objectives of this paper. This research paper provides a step-by-step procedure on the decision and designing of steering parameters for a variable Ackerman geometry. It also includes a subtle overview of the selection of an appropriate steering mechanism.

Firstly, a discussion is carried out on the methodology adopted for the designing of the steering mechanism. This includes the calculations for determining the Ackermann steering condition, turning radius estimation, space requirement calculation, trapezoidal steering system, design of variable Ackerman system, cross-checking steering effort value, minimizing bump steer, and getting the tie rod inboard point. Then the manufacturing of the steering mechanism is elaborated also its incorporation in the car is shown. Later the results obtained are discussed. Also, a stepwise method for the designing of the steering mechanism which was adapted in the manufacturing of our formula student car is explained. Additionally, a comparison of theoretical and analytical values is done. Lastly, a conclusion is made based on the study.

\section{MethodOLOGY}

The whole process of designing the Ackerman geometry is broken down into the following nine simple steps. When 
followed in order, these steps will result in a fully defined and functional variable Ackerman steering system. Lastly, the manufacturing of the steering mechanism is discussed.

\section{A. Ackermann Steering Condition}

The very slow movement of a vehicle results in a kinematic condition between the inner and outer wheels that allows a slip-free turn. This condition is termed as Ackerman condition and is usually expressed by Equation 1 .

$$
\operatorname{Cot} \delta o-\operatorname{Cot} \delta i=\frac{w}{l}
$$

Where $\delta i$ is the steering angle of the inner wheel, and $\delta o$ is the steering angle of the outer wheel.

The inner and outer wheels are defined based on the turning center $\mathrm{O}$ [10]. The Center of Mass of the steered vehicle will turn on a circle with a radius $R$, which is determined using Equation 2.

$$
R=\sqrt{a_{2}^{2}+l^{2} \operatorname{Cot}^{2} \delta}
$$

Where $\delta$ is the cot-average of the inner and outer steer angles. The cot values are calculated by utilizing Equation 3.

$$
\operatorname{Cot} \delta=\frac{\operatorname{Cot} \delta o+\operatorname{Cot} \delta i}{2}
$$

The $R$ is termed as the turning radius of the vehicle [10]. All these parameters are shown in Figure 2.

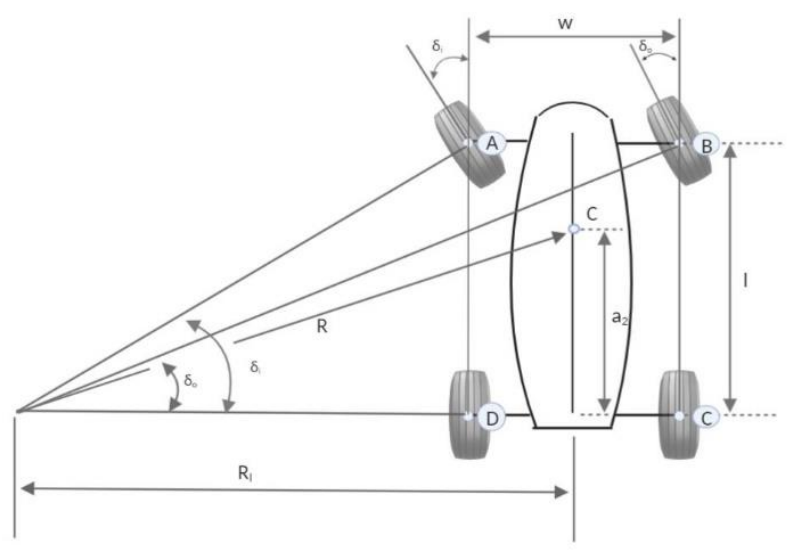

Figure 2: Figure for a vehicle turning in a Pro-Ackerman condition

A Matlab code 1 (Annexure) was generated on the equation 1,2, and 3 to find the value of turning required in one wheel concerning the other for the required Ackerman geometry.

\section{B. Turning Radius Estimation}

The Estimation of the turning radius range is another crucial yet less discussed step in designing the steering system. For a car to have the fastest corner and least lap time, there is a need to corner on the maximum turning radius possible for that turn. This is to provide maximum braking and acceleration capacity. So, all the corners on the track are studied, and the maximum possible turning radius for each corner is determined based on tire and engine specifications. The smallest value from the list of those maximum possible radii is taken and further reduced to give the vehicle some additional cornering capability. It is denoted as the least turning radius required. This provides the turning radius range for the design of any geometry.

Matlab codes 2\&3 (Annexure) were generated to determine the value of steered wheel angles and for the turning radius required to find the range of turning angle needed on the inner wheel.

\section{Space Requirement Calculation}

Calculation of the space required is necessary to ensure that the steering system opted fits the race track conditions before proceeding to further calculations. The racing track's width should always be more than the required space for the system to work as expected.

The space required can be calculated very easily by applying simple trigonometry given in Figure 3.

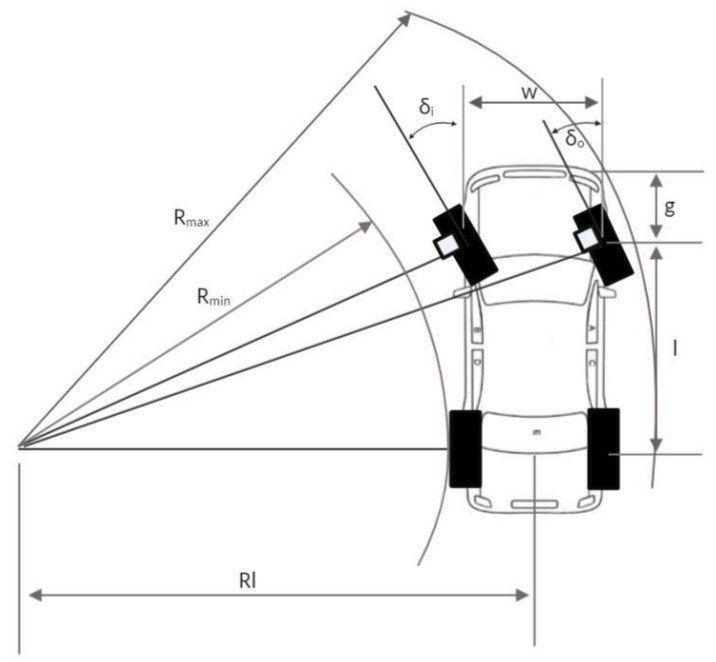

Figure 3: Figure for a fully assembled vehicle turning in Pro-Ackerman condition 
The relation obtained from the calculations is shown in Equations 4 and 5.

$\Delta R=\sqrt{\left(\frac{l}{\tan \delta i}+2 w\right)^{2}+(l+g)^{2}}-\frac{l}{\tan \delta i}$

Where,

$$
\Delta R=R_{\max }-R_{\min }
$$

$\Delta \mathrm{R}$ is the space required for the vehicle to take a turn.

A Matlab code 4 (Annexure) was designed to determine the required turning space (Annexure) and compare it with all the competition's track sizes.

\section{Trapezoidal Steering System}

Achieving an Ackerman condition at all instances in a corner is not possible by a simple mechanical method so it requires all the wheels to be steered independently [11]. Therefore, a system is designed to perform the desired Ackerman system to the closest proximity possible.

The type of steering mechanism used to achieve the Ackerman geometry required by us was a symmetric fourbar linkage, called a trapezoidal steering mechanism as shown in Figure 4.

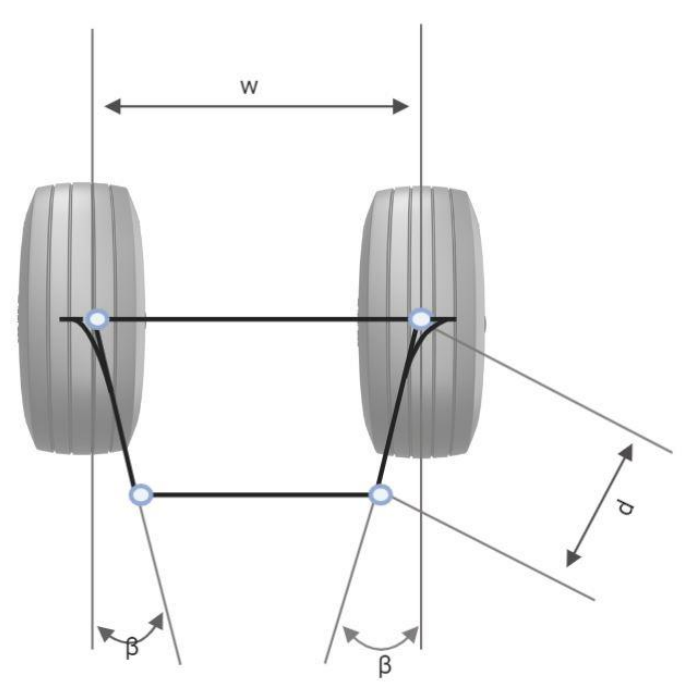

Figure 4: Schematics of trapezoidal steering Mechanism

The mechanism has two characteristic parameters: angle $\beta$ and offsets arm length $d$. A steered position of the trapezoidal mechanism is also shown in Figure 5 to illustrate the inner and outer steer angles $\delta i$ and $\delta o$.

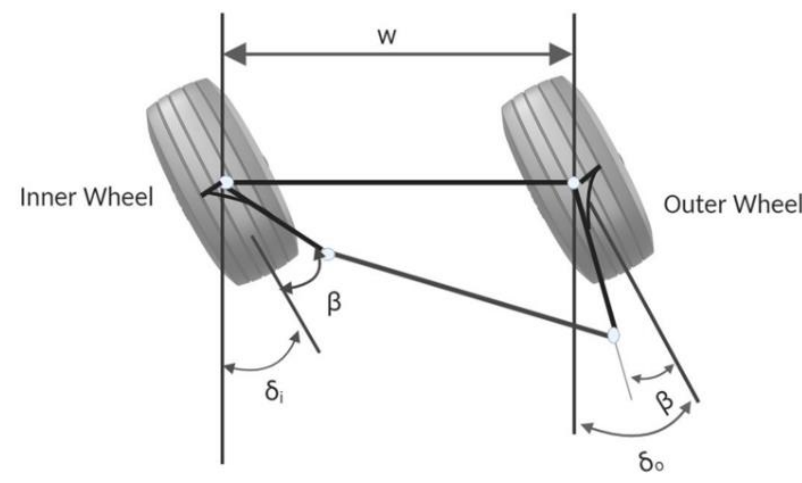

Figure 5: Schematics of the steered configuration of a trapezoid mechanism

The relationship between the inner and outer steer angles of a trapezoidal steering mechanism is

$$
\begin{gathered}
\operatorname{Sin}(\beta+\delta i)+\operatorname{Sin}(\beta-\delta o)=\frac{w}{d}+ \\
\sqrt{\left(\frac{w}{d}-2 \operatorname{Sin} \beta\right)^{2}-(\operatorname{Cos}(\beta-\delta o)-\operatorname{Cos}(\beta+\delta i))^{2}}
\end{gathered}
$$

A Matlab code 5 (Annexure) was generated based on Equation 6 [10] to obtain the values of inner and outer wheel turning angles for the system designed.

\section{E. Designing the Trapezoidal Steering System by Minimizing the RMS Error between Ackerman and Trapezoidal Systems}

Since Ackerman's condition at all the instances of a turn can't be achieved simultaneously, so the steering for the whole range of turning radius is optimized [12]. This optimization using the root mean square (RMS) error gave us the least deviation possible from the Ackerman system across the entire range of turning radius.

The concept minimized the RMS error in the values of steering required and steering achieved on the outer wheel for the same steering input on the inner wheel. This RMS method accounts for both positive and negative deviations of the angles and minimizes their values. Thus, gives the best design possible.

A Matlab code 6 (Annexure) for minimizing the RMS error was generated to determine the ideal value of $\beta$ (steering moment arm angle) and $d$ (steering moment arm length) for such a trapezoidal system. 


\section{F. Design of Variable Ackerman System}

After getting a value of $\beta$ and $d$, the outboard point for the tie rod and the steering moment arm for the system can be designed efficiently. To have multiple Ackerman benefit on the same system, the determination of various outboard points on the tie rod should be done for a different range of turning radii which can be carried out by identifying other sets of $\beta$ and $d$. The same Matlab codes are used with different input values for the process.

\section{G. Cross-Checking Steering Effort Value}

The smaller values of the steering arm length and the steering wheel travel, the more is effort required. The arm length decision considers both the factors mentioned. Once the steering arm length is selected, then the steering effort is calculated at static conditions [4]. So, the minimum and maximum steering effort value that your driver can manage is used as a boundary condition for the corresponding minimum and maximum values of steering moment arm length. Several values $d$ in this range are chosen, and the corresponding $\beta$ angle is determined, giving several Ackerman geometry points. A single moment arm containing all these points is the solution for quickly varying the Ackerman geometry [11].

\section{H. Minimizing Bump Steer}

After finalizing $\beta$ and $d$ values, the last variable remaining in the coordinates of the tie rod outboard point is its height. This variable affects suspension characteristics more than it affects the steering characteristics. So the decision of this parameter is taken based on the suspension graphs obtained from the kinematic analysis of the system. It is mainly varied to minimize the bump steer while examining the other graphs to avoid any unwanted variation of suspension parameters.

Other than suspension characteristics, it also affects the upright design and placing of the steering rack. Therefore sometimes, a little compromise is made on the performance in order to design and accommodate the systems adequately.
Adams Car was used to analyze and optimize the suspension parameters of the car [13].

\section{Getting the Tie Rod Inboard Point}

A straight line connecting the tie rod outboard point to the instantaneous center (IC) of the wheel is the domain for the inboard point. The height of the inboard point decides the height of the steering rack, so the decision made is based on the positioning of the steering rack $[13,14]$.

After placing the steering rack, the intersection of the steering rack plane and the line connecting the outboard point to IC is the inboard point.

\section{J. Material Selection and Manufacturing Process}

As the design operates on fluctuating load cycles, it is essential to select material that has a high fatigue strength and impact toughness. In a formula student race car, weight is of utmost importance, as by increasing weight more power is required to overcome the inertia of material; hence components are designed by keeping in mind the strength to weight ratio, amongst the compared materials such as mild steel, aluminum 6061, and aluminum 7075 T6, only aluminum 7075 T6 has desirable properties as mentioned.

Aluminum 7075 T6 was utilized for the steering shaft. A $20 \mathrm{~mm}$ diameter rod of Aluminium $7075 \mathrm{~T} 6$ was purchased from Perfect Metalworks, Bangalore, with a material certification bill and was turned down to $17 \mathrm{~mm}$ on the lathe machine in Materials and Manufacturing Laboratory, SMEC, VIT, Vellore. The shaft was given to GK industries, Vellore for machining the male splines on the shaft with the dimensions taken from female splines on the quick release. The shaft was then milled with a square profile to mate with bevel gear for transmitting motion and power.

EN45 material was purchased from Perfect Metalworks, Banglore for making Bevel. The material was machined by GK industries, Vellore using Gear hobbing process. The machined bevel gears were given to Murli, Chennai for case hardening at $52 \mathrm{HRC}$. Aluminium 7075-T6 material used for the casing was given to Ushtara industries, Bangalore where the job was machined on a 5 axis $\mathrm{CNC}$ machine. For manufacturing of coupler Aluminum 7075 T6 shaft of $20 \mathrm{~mm}$ 
diameter was given to GK industries, for machining keyway $\&$ splines to mate in accordance to the standard FSAE steering Rack which was purchased from KAZ racing.

For assembly firstly the deep groove ball bearings were press-fitted in the casing at Satyamoorty industry, Vellore on a 20T hydraulic press. The Bevel gears were subsequently pressed in the respective casing using the same machine. Gear meshing was done on the lathe machine in the same industry. The steering shaft and steering column were mated with bevel gear assembly at the workshop at Vellore institute of technology using a mallet. The complete assembly was secured with a help of a safety wire. The steering column was assembled with a coupler using a metal key, and the coupler was assembled with the steering column by aligning the internal splines of the coupler with splines on the steering rack. Figure 6 depicts the designed assembly of the entire steering geometry which was then used in the car.

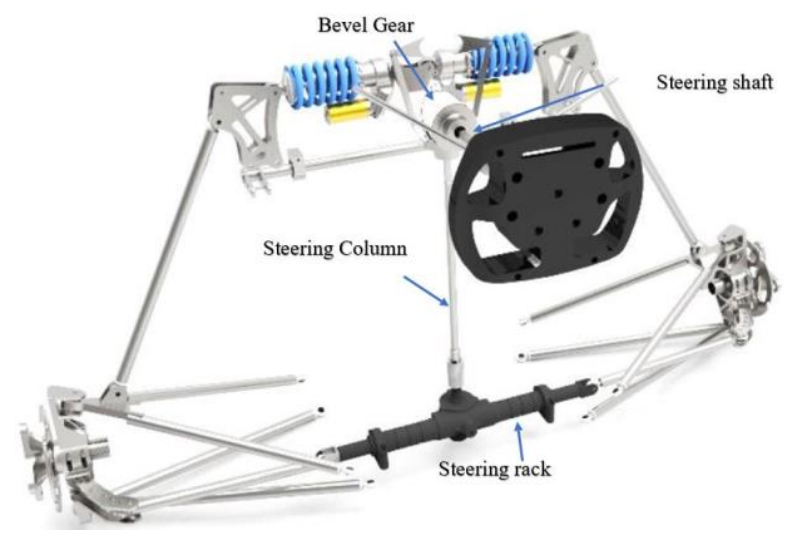

Figure 6: Schematic diagram of steering geometry assembly

\section{RESULTS}

Firstly, a stepwise process of designing the variable Ackerman geometry is generated based on the design for the steering mechanism for our car. The process is as follows:

- Selection of Pro-Ackerman

- Determination of turning radius range

- Determination of range of angle on the inner wheel for the corresponding turning radius range

- Determination of range for steering moment arm length through steering effort range
- Selection of as much number of values for steering moment arm length in the range as much variable Ackerman geometries we need

- Determination of the $\beta$ value for every corresponding steering moment arm length in the determined range of angles on inner wheel

- Repetition of the whole process from turning radius range for another set of $\beta$ and $d$ values for all the Ackerman geometries required

- Determination of height of the outboard point of tie rod

- Determination of inboard point of tie rod corresponding to the most used outboard point.

Results achieved by this designing process met our requirements, and the car PRV 18 successfully claimed many awards in Formula student Australia and Formula student Germany of the year 2018 and 2019, respectively. Some of the fundamental results are depicted below.

\section{A. System Selection}

After taking our competition track and our car's capacity into account, we finally decided to design an Ackerman steering geometry to get the most of all the tight corners and achieve an overall better performance. The results obtained for relative turning angles on inner and outer wheels using Matlab code 1(Annexure) are depicted in Figure 7.

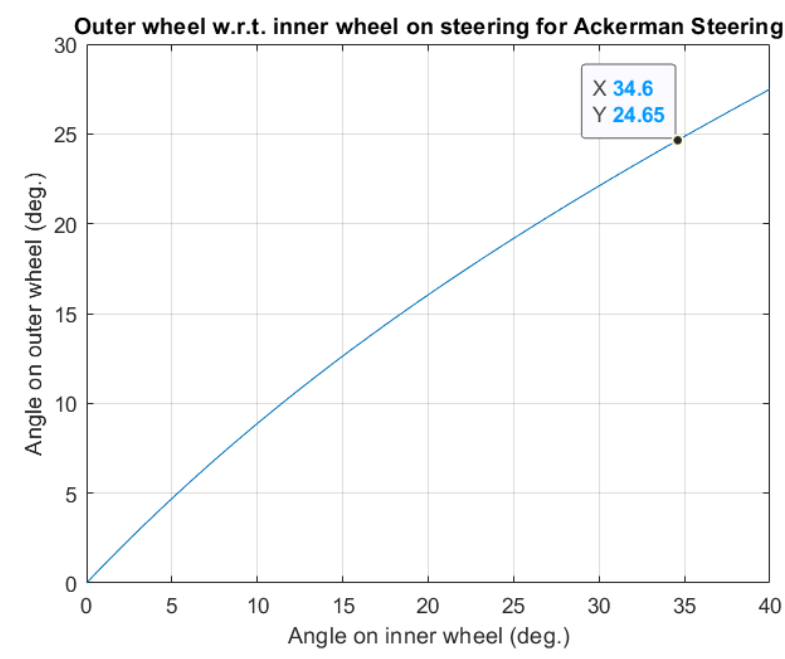

Figure 7: Graph for corresponding angles on the inner and outer wheel for Ackerman steering 


\section{B. Turning Radius Range}

The turning radius range for our car turned out to be 3000 $\mathrm{mm}$ to $\infty$ (when the vehicle is in straight-line motion). The range was sufficient enough to perform up to the car's maximum capacity.

\section{Wheel Turn Angle Range}

For the corresponding minimum turning radius i.e. 3000 $\mathrm{mm}$, the maximum inner wheel turning angle was obtained from the Matlab code and taken as $35^{\circ}$ for further calculations. This gave us the turning angle range for the inner wheel from 0 to 35 degrees, corresponding to the required turning radius range. The range was properly functional, with all the clearances satisfied.

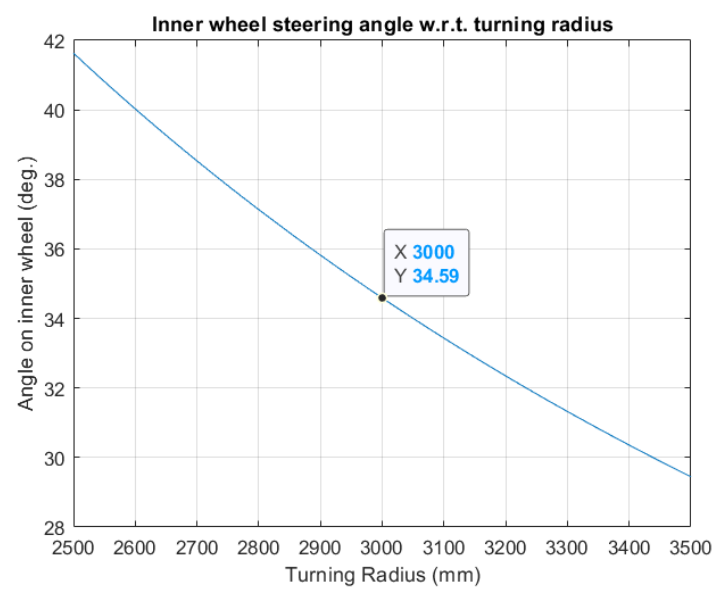

Figure 8: Graph for the angle on the inner wheel for the corresponding turning radius.

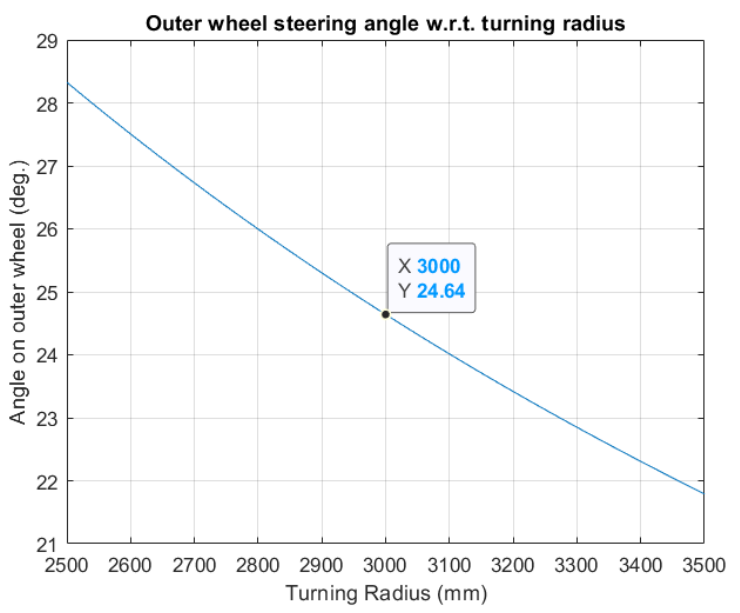

Figure 9: Graph for the angle on the outer wheel for the corresponding turning radius.
Figure 8 and Figure 9 represent the values of angle required on the inner and outer wheels for the corresponding turning radius and were obtained using Matlab code $2 \& 3$ (Annexure) respectively. Further, the values of angles on inner and outer wheels for $3000 \mathrm{~mm}$ of turning radius in figure 8 and figure 9 are the same as in figure 7 for Ackerman geometry, thus verifying that the method and the calculations followed were correct.

\section{RMS Minimization}

The value $\beta$ for the first point of Ackerman geometry was obtained as $\beta=30.14$ degrees at $d=116 \mathrm{~mm}$ with the minimum RMS value of $2.622 * 10^{-6}$. This iteration was capable of running the car in all the events of the competition, though multiple outboard points were determined for event-wise better performance.

Matlab code 6 (Annexure) was used to obtain the required length of the steering moment arm to achieve the minimum RMS error which resulted in the graph shown in Figure 10.

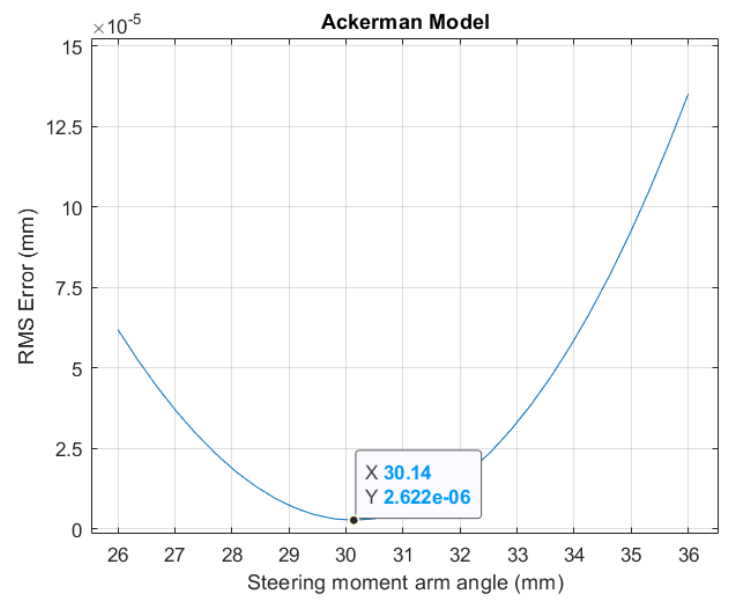

Figure 10: Graph for minimizing the RMS error plotting steering moment angle vs RMS error.

\section{E. $\quad$ Front View Suspension and Steering Geometry}

The given sketch in Figure 11 represents the front view for the suspension and steering geometry designed on Solidworks. The geometry obtained from joining the outboard point of the tie rod to IC for finding the inboard point of the tie rod gave us the minimum possible bump steer. 


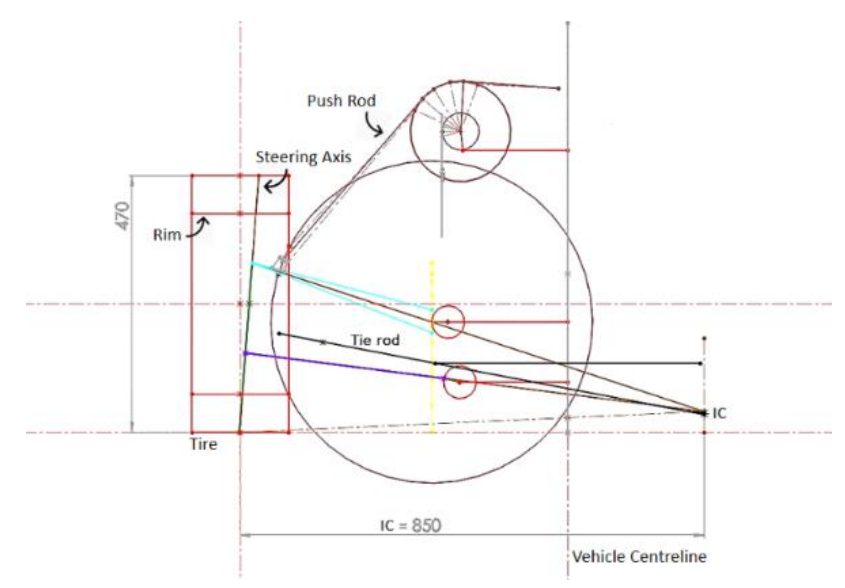

Figure 11: Front view of the design of the suspension geometry design for the formula student race car

\section{F. Bump Steer}

Adams Car was used as the kinematic analysis software for all the suspension and steering related simulations, and the obtained graph for bump steer is given in Figure 12. The bump steer obtained in bump and droop was from 0 to 0.017 degrees and 0 to 0.045 degrees respectively. The value for bump steer was negligible and hence ineffective.

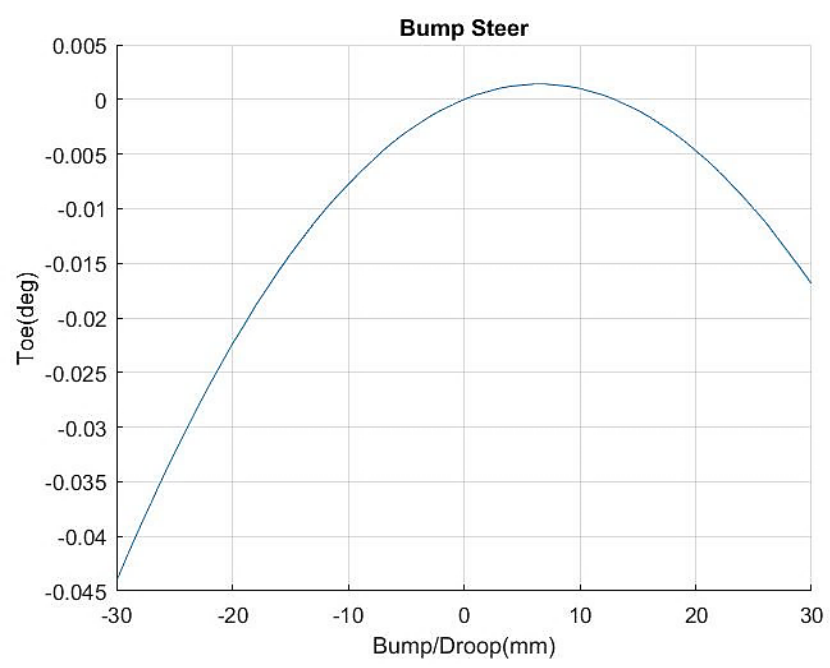

Figure 12: Graph for bump steer. Plotting bump/droop vs Toe

\section{G. Trapezoidal System}

Matlab code 5 (Annexure) was used for finding the relative turning angles on the inner and outer wheels that are achieved from the designing of the trapezoidal system and the graph obtained is shown in Figure 13. The trapezoidal system designed was very close to the required Ackerman system, and the maximum possible deviation was observed to be $1.3^{\circ}$ after comparing figure 7 and figure 13 . The difference was small enough to run the car on this system.

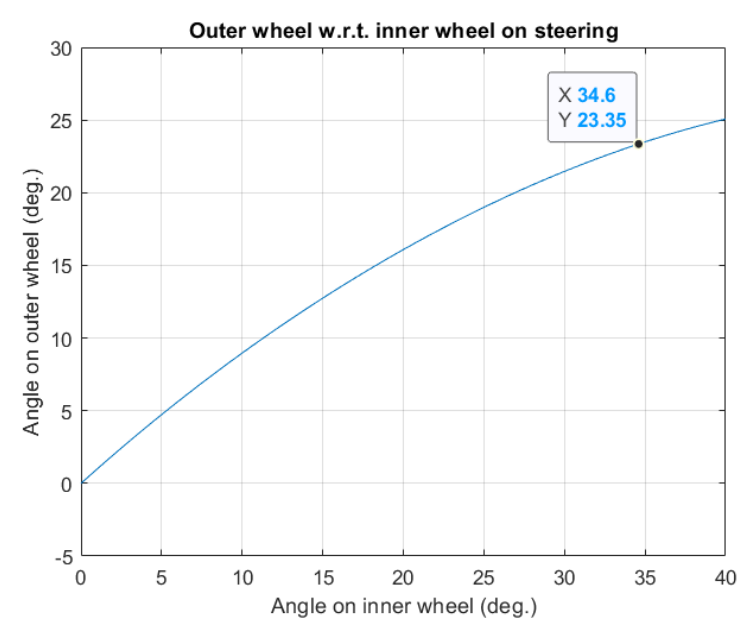

Figure 13: Graph of angles on the inner and outer wheels for the designed trapezoidal steering system.

\section{CONCLUSION}

This paper helps to determine the right choice for the selection of the steering mechanism. In this paper, a vigorous study is carried out that resulted in many graphs which were obtained based on different methods available and also based on the decision of various parameters for Ackerman geometry. After a lot of iterations and testing, we generated an effective method for the complete design of steering geometry while considering all the steering and suspension characteristics. The most important process was the calculation of RMS error that was used to ensure the minimum scrub loss. Some other methods like calculation of space required and inclusion of IC in design took care of the practical constraints and minimized the bump steer. The research, simulations, and the results obtained concluded that the proposed method turned out to be a streamlined and precise way for the decision and designing of a variable Ackerman steering geometry. In the future inclusion of sixbar calculations and slip, angles can make the method more accurate and useful.

\section{REFERENCES}

[1] Belkhode, P.N., Washimkar, P.V., and Dhande, M.S., "Prdication of Steering Geometry of Front Suspension using Experimental Data-Based Model," IJET 2(6):543-546, 2010, DOI:10.7763/IJET.2010.V2.179. 
[2] Milliken, W.F. and Milliken, D.L., "Race car vehicle dynamics," Society of Automotive Engineers, Warrendale, Pa., ISBN 1560915269, 1995.

[3] Deng Lili, X.H., "Optimization of Race Car Divided Steering Trapezium: 9 - 11 Sept. 2011, Ningbo, China; proceedings," IEEE, Piscataway, NJ, ISBN 9781457703218, 2011.

[4] Dhiraj Malu, Nikhil Katare, Suraj Runwal, Saurabh Ladhe, "Design Methodology for Steering System of an ATV.," International Journal of Mechanical Engineering and Technology, 7(5), pp. 272-277, 2016.

[5] Nitish Malik, Prakhar Agarwal \& Ajay Rajput, "Design and Performance Optimization of the Steering System of a Vehicle," Trans Stellar, 2017. DOI: 10.24247/ijauerddec 20171

[6] Biswal, S., Prasanth, A., Udayakumar, R., Sankaram, M.N. et al., "Design of steering system for a small Formula type vehicle using tire data and slip angles," MATEC Web Conf. 124:7007, 2017, DOI:10.1051/matecconf/201712407007.

[7] Chinmay Raut, Rahul Chettiar, Nikunj Shah, Chandraprakash Prasad, Dhiraj Bhandarkar, "Design, Analysis and Fabrication of Steering System Used in Student Formula Car," Volume 5, Issue V, International Journal for Research in Applied Science and Engineering Technology (IJRASET) Page No: 15291540, 2017.

[8] Nikhil N. Gitay, Siddhart A Joshi, Ajit A Dumbre, Devesh C Juvekar, "Design \& Manufacturing of an Effective Steering System for a Formula Student Car," International Journal of Innovative Research in Technology,8(4), 2018.

[9] Naveen, T. Deepak Varma, D. Sudhakar Reddy, N. Guru Vardhan, and K. Chandra Mouli, "Design Of Steering Geometry For Formula Student Car's,” International Journal of Mechanical Engineering and Technology (IJMET) Volume 9, Issue 5, pp. 182-192, 2018.

[10] Jazar, R.N., "Vehicle dynamics: Theory and application," Springer, New York, ISBN 1461485436, 2014.

[11] Smith, C., "Tune to win: The art and science of race car development and tuning," Motorbooks International, Redwood City, Calif., ISBN 0879380713, 1999.

[12] Gillespie, T.D., "Fundamentals of vehicle dynamics," Society of Automotive Engineers, Warrendale, PA, ISBN 1560911999, 1992.
[13] Andrew S. Ansara, Andrew M. William, Maged A. Aziz, Peter N. Shafik, "Optimization of Front Suspension and Steering Parameters of an Off-road Car using Adams/Car Simulation," International Journal of Engineering and Technology (IJERT) Volume 06, Issue 09, 2017.

[14] Kelkar, S.S., Gautam, P., Sahai, S., Agrawal P.S., Manoharan R. A detailed study on design, fabrication, analysis, and testing of the anti-roll bar system for formula student cars. SN Appl. Sci. 3, 302, 2021. https://doi.org/10.1007/s42452-02104279-z

\section{ANNEXURE}

\section{Matlab Code 1:}

Relation between inner and outer wheel angles in an Ackerman geometry

clc;

clear all

$1=1600 ; \quad \%$ l=wheelbase of car

$\mathrm{w}=1200-32 ; \% \mathrm{w}=$ trackwidth of car

$\operatorname{did}=0: 0.1: 40 ; \% \mathrm{did}=$ inner wheel turning angle in degrees for $\mathrm{i}=1$ :length(did)

$$
\begin{aligned}
& \operatorname{di}(i)=\operatorname{did}(i) *(\operatorname{pi} / 180) ; \\
& \operatorname{cotdo}(i)=(w / l)+(\cot (\operatorname{di}(i))) ; \\
& \operatorname{do}(i)=\operatorname{acot}(\operatorname{cotdo}(i)) ; \\
& \operatorname{dod}(i)=\operatorname{do}(i) *(180 / p i) \quad \% \text { dod }=\text { outer wheel turning angle }
\end{aligned}
$$
in degrees

end

plot(did,dod)

title('Outer wheel w.r.t. inner wheel on steering for Ackerman Steering')

xlabel('Angle on inner wheel (deg.)')

ylabel('Angle on outer wheel (deg.)')

grid on

\section{Matlab Code 2:}

Relation between inner wheel angles and turning radius in an Ackerman geometry

clc;

clear all

$\mathrm{a}=752 ; \quad \% \mathrm{a}=\mathrm{CG}$ distance from rear axle 


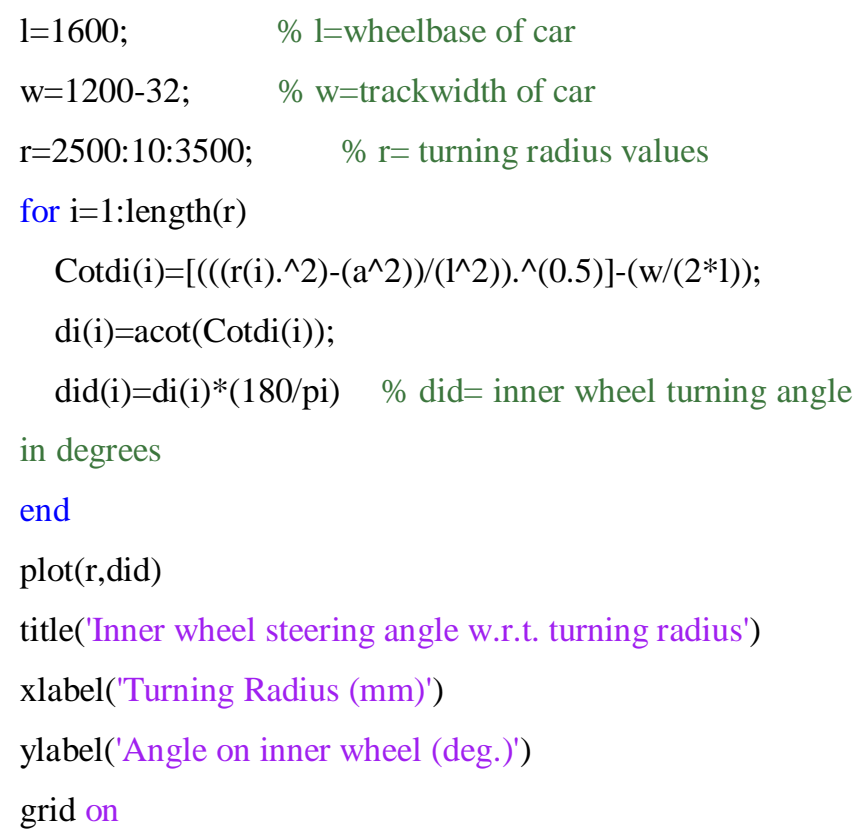

\section{Matlab Code 3}

Relation between outer wheel angles and turning radius in an ackerman geometry

clc;

clear all

$\mathrm{a}=752 ; \quad \% \mathrm{a}=\mathrm{CG}$ distance from rear axle

l=1600; \% l=wheelbase of car

$\mathrm{w}=1200-32 ; \quad \% \mathrm{w}=$ trackwidth of $\mathrm{car}$

$\mathrm{r}=2500: 10: 3500 ; \% \mathrm{r}=$ turning radius values

for $\mathrm{i}=1$ :length(r)

$\operatorname{Cotdo}(\mathrm{i})=\left[\left(\left(\left(\mathrm{r}(\mathrm{i}) .^{\wedge} 2\right)-\left(\mathrm{a}^{\wedge} 2\right)\right) /\left(\mathrm{l}^{\wedge} 2\right)\right) .^{\wedge}(0.5)\right]+(\mathrm{w} /(2 * 1)) ;$

$\operatorname{do}(\mathrm{i})=\operatorname{acot}(\operatorname{Cotdo}(\mathrm{i}))$;

$\operatorname{dod}(\mathrm{i})=\operatorname{do}(\mathrm{i}) *(180 / \mathrm{pi}) \% \operatorname{dod}=$ outer wheel turning angle

in degrees

end

$\operatorname{plot}(\mathrm{r}, \mathrm{dod})$

title('Outer wheel steering angle w.r.t. turning radius')

xlabel('Turning Radius (mm)')

ylabel('Angle on outer wheel (deg.)')

grid on

\section{Matlab Code 4}

Space required for the car to take a turn clc;

clear all $\mathrm{g}=855.1 ; \% \mathrm{~g}=$ distance between front most part to front axle

$1=1600 ; \% 1=$ wheelbase of car

$\mathrm{w}=1200-32 ; \quad \% \mathrm{w}=$ trackwidth of $\mathrm{car}$

did=0:0.01:40; \% did= inner wheel turning angle in degrees

for $\mathrm{i}=1$ :length(did)

$\operatorname{di}(\mathrm{i})=\operatorname{did}(\mathrm{i}) *(\mathrm{pi} / 180)$;

$\mathrm{x}(\mathrm{i})=((\mathrm{l} /(\tan (\operatorname{di}(\mathrm{i}))))+2 * \mathrm{w})$;

$y(i)=1+g$;

$\operatorname{dr}(\mathrm{i})=\left((\mathrm{x}(\mathrm{i})) \cdot{ }^{\wedge} 2+(\mathrm{y}(\mathrm{i})) \cdot{ }^{\wedge} 2\right) \cdot^{\wedge}(0.5)-\mathrm{l} /(\tan (\operatorname{di}(\mathrm{i}))) \quad \%$ space required by vehicle make the turn

end

plot(did,dr)

title('Space required w.r.t. inner wheel steering angle')

xlabel('Inner wheel angle (deg.)')

ylabel('Turning space required (mm)')

grid on

\section{Matlab Code 5:}

Relation between inner and outer wheel angles in trapezoidal system

clc;

clear all

$1=1600 ; \quad \% 1=$ wheelbase of car

$\mathrm{t}=1200-32 ; \quad \% \mathrm{t}=$ trackwidth-kingpin offset

$\mathrm{d}=116 ; \% \mathrm{~d}=$ steering moment arm length

beta $=30.25 * \mathrm{pi} / 180 ; \%$ beta $=$ steering moment arm angle in degrees

$\operatorname{did}=0: 0.1: 40$;

syms do real;

for $\mathrm{i}=1$ :length(did)

$\operatorname{di}(\mathrm{i})=\operatorname{did}(\mathrm{i}) *(\mathrm{pi} / 180)$;

eq $(\mathrm{i})=\left(\mathrm{t}-2 * \mathrm{~d}^{*} \sin (\text { beta })\right)^{\wedge} 2==(\mathrm{t}-\mathrm{d} * \sin ($ beta $+\mathrm{di}(\mathrm{i}))-$

$\mathrm{d}^{*} \sin ($ beta-do $\left.)\right)^{\wedge} 2+\left(\mathrm{d}^{*} \cos (\text { beta-do })-\mathrm{d}^{*} \cos (\text { beta }+\mathrm{di}(\mathrm{i}))\right)^{\wedge} 2$; $\operatorname{dod}(\mathrm{i})=\operatorname{vpasolve}(\mathrm{eq}(\mathrm{i}), \mathrm{do})$;

end

$\operatorname{dod}=\operatorname{vpa}(180 * \mathrm{dod} / \mathrm{pi})$

plot(did,dod)

title('Outer wheel w.r.t. inner wheel on steering')

xlabel('Angle on inner wheel (deg.)')

ylabel('Angle on outer wheel (deg.)')

grid on 


\section{Matlab Code 6}

\section{Minimizing of RMS Error}

clc

clear all

close all

$\mathrm{xi}=$ linspace $(1,26,50) ; \quad \% \mathrm{xi}=$ range of inner wheel angles

$\mathrm{l}=1.6 ; \mathrm{a}=0.53 * \mathrm{l} ; \mathrm{b}=0.47 * 1 ; \mathrm{t}=1.2-.032 ; \% \mathrm{l}=$ wheelbase of car in meters

$\mathrm{d}=0.116 ; \quad \% \mathrm{~d}=$ steering moment arm length

beta=linspace $(26,36,10) ; \quad \%$ beta $=$ steering moment arm

angle

xo=zeros(length(beta),length(xi));

syms yo

for $\mathrm{i}=1$ :length(beta)

for $\mathrm{j}=1$ :length(xi)

$x o(i, j)=\operatorname{acot}((t / l)+\cot (x i(j) * p i / 180))$;

$\mathrm{eq}(\mathrm{i}, \mathrm{j})=(\mathrm{t}-2 * \mathrm{~d} * \sin (\operatorname{beta}(\mathrm{i}) * \mathrm{pi} / 180))^{\wedge} 2==(\mathrm{t}-$

d* $\sin (($ beta(i)+(xi(j)))*pi/180)-d*sin(beta(i)*pi/180-

yo $))^{\wedge} 2+\left(\mathrm{d}^{*} \cos (\right.$ beta(i)*pi/180-yo)-

$\left.d^{*} \cos \left((\operatorname{beta}(\mathrm{i})+\mathrm{xi}(\mathrm{j}))^{*} \mathrm{pi} / 180\right)\right)^{\wedge} 2$;

end

end

for $\mathrm{i}=1$ :length(beta)

for $\mathrm{j}=1$ :length(xi)

$$
y(i, j)=v p a s o l v e(e q(i, j), y o) ;
$$

end

end

for $\mathrm{i}=1$ :length(beta)

for $\mathrm{j}=1$ :length(xi)

$$
\mathrm{z}(\mathrm{i}, \mathrm{j})=(\mathrm{y}(\mathrm{i}, \mathrm{j})-\mathrm{xo}(\mathrm{i}, \mathrm{j})) \cdot \wedge 2
$$

end

end

$\mathrm{w}=(\operatorname{sum}(\mathrm{z}, 2)) /$ length $(\mathrm{xi})$;

plot(beta, w');

title('Ackerman Model')

xlabel('Steering moment arm angle (mm)')

ylabel('RMS Error (mm)')

grid on

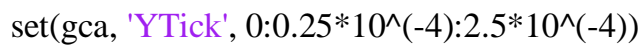

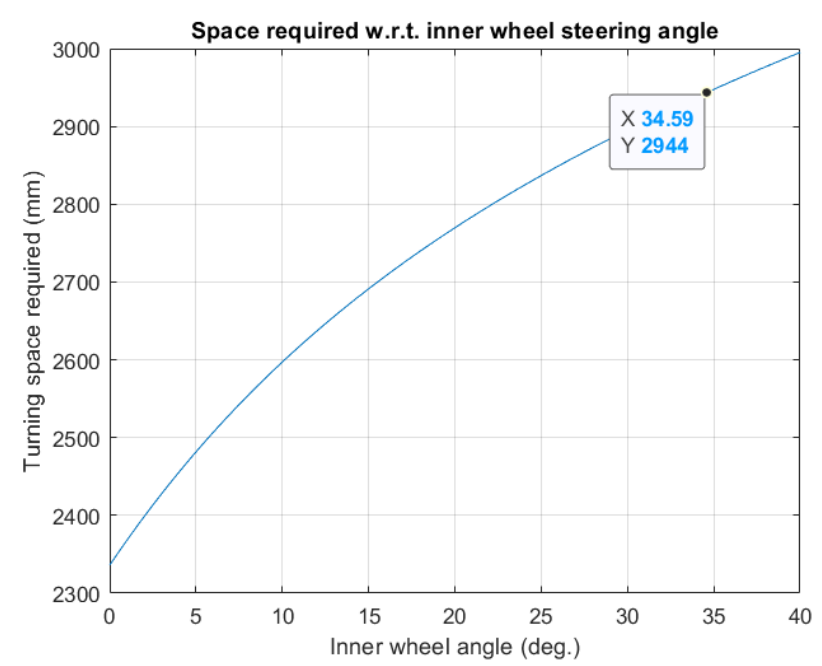

Figure 14: Graph of Inner wheel angle vs Turning Space required 